\title{
Nonlinear measures in posturography compared to linear measures based on yoga poses performance
}

\author{
MICHALINA BŁAŻKIEWICZ*
}

Józef Piłsudski University of Physical Education, Warsaw, Poland.

\begin{abstract}
Purpose: Yoga is known as a type of exercise that combines physical, mental and spiritual aspects. There has not been much research on the postural control in various yoga poses. The aim of this study was to examine CoP regularity in both yoga instructors and novices during the performance of four yoga poses, and to verify the sensitivity of linear and nonlinear methods for assessing postural stability. Methods: The dynamic characteristics of CoP fluctuations were examined using linear and nonlinear methods on a group of 22 yoga instructors (Y) and 18 age-matched non-practitioners of yoga (NY). The study involved maintaining a balance for 20 seconds while performing four yoga poses. Results: Conventional analysis of CoP trajectories showed that NY and Y exhibited similar control of postural sway, as indicated by similar CoP path-length and area values observed in both groups. These results suggest that the special balance yoga training received by $\mathrm{Y}$ may not have an impact on less challenging balance conditions, such as the poses used in this experiment. Interestingly, nonlinear dynamical analysis of CoP showed that $\mathrm{Y}$ exhibited less CoP regularity and more complex signal than NY, as evidenced by higher values of sample entropy and fractal dimension. Conclusions: The results shed light on the surplus values of $\mathrm{CoP}$ trajectories in the nonlinear dynamical analysis to gain further insight into the mechanisms involved in posture control.
\end{abstract}

Key words: postural control, center of pressure, yoga, complexity, regularity

\section{Introduction}

Various indicators of postural control in humans have been studied for decades, mostly based on the trajectory of the center of pressure (CoP). Traditional approaches focus on CoP variability based on the notion that during standing still it displays high irregularities and non-stationary fluctuations. Delving deeper, in order to understand the physiology of postural control, the $\mathrm{CoP}$ signal evaluation techniques can be divided into: linear and nonlinear measures [4], [18].

Linear tools, such as the CoP path length, sway velocity and area, quantify the amount of $\mathrm{CoP}$ movement during a specific task, independently of their order in the distribution. The nonlinear system approach helps to evaluate different aspects of the CoP data. Nonlinear measures are able to capture the temporal component of the movement variation in $\mathrm{CoP}$ with regards to how motor behavior develops over time. Thus, these measures allow to quantify regularity, complexity, and stability [4], [18], [21]. Nonlinear tools include the largest Lyapunov and Hurst exponents, as well as fractal dimension and entropy families.

In order to use nonlinear measures, it is necessary to demonstrate the presence of chaos in the system. One of the basic tools used to identify chaos is the presence of the largest positive Lyapunov exponent [12]. The Hurst exponent is another measure that confirms the presence of chaos in the system. It quantifies the fractal properties of a time series and its long-term memory [7]. Higuchi [9] came up with a relatively

\footnotetext{
* Corresponding author: : Michalina Błażkiewicz, Józef Piłsudski University of Physical Education, ul. Marymoncka 34, 00-968, Warsaw, Poland. Phone: 503121114, e-mail: michalinablazkiewicz@gmail.com

Received: April 16th, 2020

Accepted for publication: July 16th, 2020
} 
straight-forward technique that can be applied to time series data to extract the fractal dimension (FD). When applied to CoP displacement, a FD result should fall between 1 (no variability) and 2. In the case of the CoP trajectory, a change in FD may indicate a change in control strategies for maintaining quiet stance. Doyle et al. [6] concluded that fractal analysis represents a reliable method of highlighting the specific characteristics of balance control, in contrast to traditional measures. The entropy family quantifies signal regularity. Sample entropy (SEn) is one of the types of entropy measures. Its advantage is independence of data length [17]. The increased values of SEn, which indicate larger irregularity of the CoP, have been attributed to a reduced amount of attention invested in posture [18] and may be interpreted as an increase in the efficiency or 'automaticity' of postural control [4]. Lower SEn is associated with less complexity or more regular physical movement [4]. As complexity is crucial to flexibility in adapting to one's surroundings, the lower complexity of physical movement translates to lower flexibility and higher rigidity for postural control. Conversely, higher SEn, which is increased complexity, is interpreted as increased self-organization and effective strategy in postural control [4]. For instance, the SEn has been used to investigate the effect of visual perception [20], cerebral concussion [3] and cognitive tasks on postural dynamics [1], [15]. In summary, nonlinear analyses of the CoP data can provide a window into the neurological state of the human, and allow for insight into the multifaceted strategies utilized in movement and posture.

A good example of combining physical fitness with the nervous and physiological systems can be found, it seems, in yoga practice. Yoga is known as a type of exercise that combines physical, mental and spiritual aspects. According to the yoga encoder, an asana is any posture that remains steady and comfortable [23]. Asanas are also defined as isometric exercises, which involve a coordinated action of synergist and antagonist muscles, requiring flexibility and precision in body movement. The advantages of yoga practice have been widely studied [25]. Some studies showed improvements in well-being, anxiety, stress, balance and gait parameters after practicing yoga [11]. However, there are no studies investigating the linear and nonlinear parameters to assess the postural control complexity during standing in the most common yoga asanas. Therefore, the aim of this study was to examine $\mathrm{CoP}$ regularity in instructors and novices during the performance of four yoga poses, and to verify additional usage of linear and nonlinear methods for assessing postural stability.

\section{Materials and methods}

\subsection{Participants}

Two groups, Y and NY, participated in the study (Table 1). Group Y consisted of twenty two female yoga instructors with a mean experience of $2 \pm 0.5$ years. Group NY consisted of eighteen women who have never practiced yoga.

Table 1. Participant characteristics (means and standard deviations values), where: $\mathrm{Y}$ - group of yoga instructors, $\mathrm{NY}$ - group of non-practicing yoga women

\begin{tabular}{|c|c|c|c|}
\hline Group & $\begin{array}{c}\text { Age } \\
{[\text { years }]}\end{array}$ & $\begin{array}{c}\text { Body weight } \\
{[\mathrm{kg}]}\end{array}$ & $\begin{array}{c}\text { Body height } \\
{[\mathrm{cm}]}\end{array}$ \\
\hline $\mathrm{Y}(n=22)$ & $28.5 \pm 2.8$ & $59.7 \pm 8.5$ & $165 \pm 0.7$ \\
\hline NY $(n=18)$ & $21.8 \pm 5.1$ & $62.5 \pm 8.1$ & $169.5 \pm 0.6$ \\
\hline
\end{tabular}

The study was conducted according to the ethical guidelines and principles of the Declaration of Helsinki. Participants reported having no balance disorders, no vision defect and no existing lower limb injuries.

\subsection{Procedure}

Participants underwent balance measurements while performing four yoga poses (Fig. 1) in the following order: Standing Knee Hug, Tree, Eagle and King-Dancer.
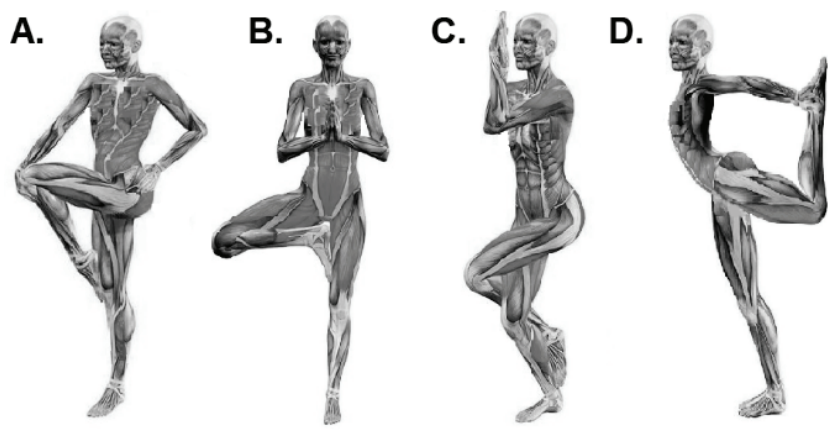

Fig. 1. Yoga poses: A. Standing Knee Hug, B. Tree, C. Eagle, D. King-Dancer

Postural stability data of the poses were obtained using an AMTI AccuSway Force Platform and the software Balance Clinic (Advanced Mechanical Technology Inc., USA). The CoP trajectories were collected 
at a sampling rate of $100 \mathrm{~Hz}$. Participants stood barefoot on the platform and each attempt lasted 20-seconds. There was a 2-minute break between each pose. In selected yoga pose, data collection began after participants stated they felt stable which was signaled by them with a short beep without opening the mouth.

\subsection{Data analysis}

For the purpose of our study, we selected the most common parameters involving linear and nonlinear methods, as proposed by various authors [13], [18]. Two linear parameters: CoP path length and area of ellipse were taken for further analysis. Both parameters were extracted directly from Balance Clinic software. Measurement of the CoP path length involves computing the distance covered by the CoP over a certain time period by summing the Euclidean distances between points: CoP path length $=\sum_{i=2}^{n} \sqrt{\left(x_{i}-x_{i-1}\right)^{2}+\left(y_{i}-y_{i-1}\right)^{2}}$, where $x, y$ are the coordinates of the $\mathrm{CoP}$ and $n$ is the number of data points. The area of ellipse is the surface area given by the $95 \%$ confidence ellipse representing the smallest ellipse that covers $95 \%$ of the points of the postural sway. The area of ellipse is computed using the eigenvalues of the variance/covariance matrix. Moreover, raw CoP data from the force plate were analyzed using MatLab software (MathWorks, USA). In order to examine whether the posture is actively controlled in the direction of largest postural sway [18], both the registered medio-lateral (ML) and anterio-posterior (AP) CoP data were analyzed. In addition, for nonlinear parameters, we used the mean value from two directions (AP-ML). Four nonlinear methods were used to assess CoP dynamics by means of sample entropy, largest Lyapunov exponent, fractal dimension and Hurst exponent, which are briefly explained in the following section. Next, all parameters were tested for normal distribution. The two-factor ANOVA was used in order to find differences between the groups. The factors were: 1) position and 2) group membership (NY or Y). Next, using the Tukey's post-hoc test, significant effects for the individual linear and nonlinear parameters were identified. All statistical analyses were performed using Statistica v.13 (StatSoft. Inc., USA), with a significant $p$-value set at 0.05 .

\section{Sample entropy (SEn)}

SEn evaluates the regularity of a given time-series. SEn calculates the probability that a sequence of $N$-data points, having repeated itself within a tolerance $r$ for $m$ points, may also repeat itself for $m+1$ points, without allowing self-matches: $\operatorname{SEn}(m, r, N)=-\ln \left(\frac{A^{m}(r)}{B^{m}(r)}\right)$.

$B$ represents the total number of matches of length $m$ while $A$ represents the subset of $B$ that also matches for $m+1$. Thus, a low SEn value arising from a high probability of repeated template sequence in the data means greater regularity. For calculating the SEn, we used the MatLab codes obtained from Physionet tool [8] and the "default" parameters: $m=2, r=0.2$.

\section{Largest Lyapunov exponent ( $\lambda$ )}

This measure is a gauge of the local stability of a system, i.e., its resistance to small internal perturbations, such as the natural fluctuations that occur while maintaining an upright stance [19]. The idea of using $\lambda$ to identify chaos in a system is based on the assumption that if the average distance between two points increases at the exponential rate, the system is sensitive to a change in initial conditions, and the value of $\lambda$ is greater than zero. Thus, $\lambda$ can be defined using the following equation: $d(t)=C e^{\lambda t}$, where: $d(t)$ is the average divergence at time $t$ and $C$ is a constant that normalizes the initial separation [16]. Therefore, the existence of a positive $\lambda$ is often considered a necessary and sufficient condition for the presence of chaos in the system.

\section{Fractal Dimension (FD)}

It can be used to estimate the dimensional complexity of time-series. The FD was calculated using Higuchi's algorithm [9], which was written in Matlab. Higher FD values are associated with greater complexity of the CoP path, with lower FD values indicative of a less complex (or "straighter") $\mathrm{CoP}$ path.

\section{Hurst exponent (H)}

The Hurst exponent indicates the presence or absence of (long-range) correlations [10]. Applied to CoP data, the $\mathrm{H}$ can be interpreted as a measure of roughness. The larger the value of $\mathrm{H}$, the smoother the time-series. $\mathrm{H}$ is bounded to the interval $(0,1)$. The properties of $\mathrm{H}$ can be summarized as follows: (1) $H=0.5$ indicates random series (Brownian process); (2) $0.5<H<1$ indicates persistent long-range power-law correlations; (3) $0<H<0.5$ indicates antipersistent (short-term memory, anti-correlated) process [24].

At this stage, a significant difference can be seen between the linear and nonlinear parameters. Linear parameters, mainly CoP ellipse area, combine data 
from the $x$ and $y$ directions and give a one-dimensional result, while nonlinear parameters must be considered as a variable in two planes separately.

\section{Results}

ANOVA yielded significant main effects of group $\mathrm{Y}$ and NY: $\mathrm{F}(14,139)=2.31, p=0.0070$ and four poses: $\mathrm{F}(42,413)=1.71, p=0.0047$. No significant effects of two factors: group $\mathrm{x}$ position interaction were found: $\mathrm{F}(42,413)=0.85, p=0.7413$.

\subsection{Effects of group $Y$ and NY}

Using the post-hoc Tukey's test, significant effects were found for nonlinear measures (Table 2): SEn and FD in each direction separately and in combination. In the Y group, almost all values of nonlinear measures were higher, compared to those obtained in the NY group.

SEn is significantly higher in each direction in the $\mathrm{Y}$ group, which suggests that the $\mathrm{CoP}$ fluctuations are much more irregular compared to the NY group. The biggest fluctuations are observed in the ML direction. FD is a measure that estimates the dimensional complexity of biomedical time-series. In the $\mathrm{Y}$ group, the FD values were significantly higher than in the NY group in both directions. This result suggests that the CoP signal is much more complex for people that practice yoga. The Hurst exponent is another nonlinear parameter for which the values were in the range of $0.75-0.82$. This result indicates persistent long-range power-law correlations, which means that the CoP series for the poses being studied does not change.

As far as the linear parameters are concerned, no significant differences were found between groups $\mathrm{Y}$ and NY for the CoP path length and the ellipse surface area. Although they were slightly higher in the $\mathrm{Y}$ group.
Table 2. The mean and standard deviation for nonlinear and linear measures calculated for all yoga poses,

for which statistically significant differences were not found and were detected $\left({ }^{*} p<0.05\right)$, where: $\mathrm{Y}-$ yoga group,

$\mathrm{NY}$ - not practicing yoga group, SEn - sample entropy, $\mathrm{FD}$ - fractal dimension, $\mathrm{H}$ - Hurst exponent, $\lambda$ - Lyapunov exponent

\begin{tabular}{|l|c|c|c|}
\hline \multicolumn{1}{|c|}{ Parameter } & $\mathrm{Y}$ & $\mathrm{NY}$ & $p$-value \\
\hline SEn (AP) & $0.42 \pm 0.13$ & $0.35 \pm 0.10$ & $0.0001^{*}$ \\
\hline SEn (ML) & $0.51 \pm 0.12$ & $0.47 \pm 0.10$ & $0.0010^{*}$ \\
\hline SEn (AP-ML) & $0.47 \pm 0.12$ & $0.41 \pm 0.10$ & $0.0001^{*}$ \\
\hline FD (AP) & $1.67 \pm 0.06$ & $1.62 \pm 0.06$ & $0.0001^{*}$ \\
\hline FD (ML) & $1.71 \pm 0.06$ & $1.68 \pm 0.05$ & $0.0014^{*}$ \\
\hline FD (AP-ML) & $1.69 \pm 0.06$ & $1.66 \pm 0.06$ & $0.0001^{*}$ \\
\hline$H(\mathrm{AP})$ & $0.75 \pm 0.05$ & $0.76 \pm 0.05$ & 0.4381 \\
\hline$H$ (ML) & $0.80 \pm 0.03$ & $0.82 \pm 0.42$ & $0.0270^{*}$ \\
\hline$H$ (AP-ML) & $0.78 \pm 0.04$ & $0.79 \pm 0.23$ & 0.1178 \\
\hline$\lambda$ (AP) & $1.10 \pm 0.22$ & $1.11 \pm 0.18$ & 0.8581 \\
\hline$\lambda$ (ML) & $1.06 \pm 0.23$ & $1.06 \pm 0.20$ & 0.8758 \\
\hline$\lambda($ AP-ML) & $1.08 \pm 0.22$ & $1.09 \pm 0.19$ & 0.8373 \\
\hline CoP path length [cm] & $99.47 \pm 26.37$ & $95.32 \pm 17.81$ & 0.2449 \\
\hline CoP area [cm $\left.{ }^{2}\right]$ & $8.67 \pm 3.28$ & $8.84 \pm 2.80$ & 0.7115 \\
\hline
\end{tabular}

\subsection{Effects of yoga poses}

Using the post-hoc Tukey's test, significant differences were found for nonlinear measures only for $\lambda$ (Table 3 ). There were statistically significant differences $(p<0.05)$ between each pose (Standing Knee Hug, Tree and Eagle) and the King-Dancer. The same results were obtained for linear measures.

Positive $\lambda$ in each yoga posture indicates that the body's balance control system is not a regular dynamic system. It means that there are no certain frequencies and regular predictable behaviors, and it has a large number of dominant frequencies. Large fluctuations are present for the King-Dancer pose, which is the most difficult, confirmed by large values of CoP path length and $\mathrm{CoP}$ area.

Table 3. The mean and standard deviation values of the Lyapunov exponent and linear parameters for yoga poses calculated in $\mathrm{Y}$ and NY group, in the sagittal (AP) and front plane direction (ML) and their combinations (AP-ML), $p-$ mean value, $p<0.05$

\begin{tabular}{|l|c|c|c|c|c|}
\hline \multicolumn{1}{|c|}{ Parameter } & Standing Knee Hug & Tree & Eagle & King-Dancer & $p$-value \\
\hline$\lambda(\mathrm{AP})$ & $1.09 \pm 0.02$ & $1.03 \pm 0.02$ & $1.08 \pm 0.02$ & $1.22 \pm 0.02$ & 0.0075 \\
\hline$\lambda(\mathrm{ML})$ & $1.07 \pm 0.03$ & $0.99 \pm 0.03$ & $1.05 \pm 0.03$ & $1.14 \pm 0.03$ & 0.0057 \\
\hline$\lambda(\mathrm{AP}-\mathrm{ML})$ & $1.08 \pm 0.03$ & $1.02 \pm 0.02$ & $1.07 \pm 0.02$ & $1.18 \pm 0.02$ & 0.0003 \\
\hline CoP path length $[\mathrm{cm}]$ & $93.25 \pm 3.57$ & $92.12 \pm 3.57$ & $95.35 \pm 3.57$ & $107.86 \pm 3.57$ & 0.0164 \\
\hline CoP area $\left[\mathrm{cm}^{2}\right]$ & $8.38 \pm 0.47$ & $7.89 \pm 0.47$ & $8.51 \pm 0.47$ & $10.08 \pm 0.47$ & 0.0067 \\
\hline
\end{tabular}




\subsection{Differences within the group}

Analyzing the differences in stability within the NY and $\mathrm{Y}$ groups, statistically significant differences were found $(p<0.05)$ for CoP path length, when comparing each pose against the King-Dancer pose. Arranging the poses from the most to the least stable resulted in the same order in both groups: Tree, Standing Knee Hug, Eagle and King-Dancer. When analyzing the average SEn values (AP-ML), the same pose order was obtained for both groups (Fig. 2).

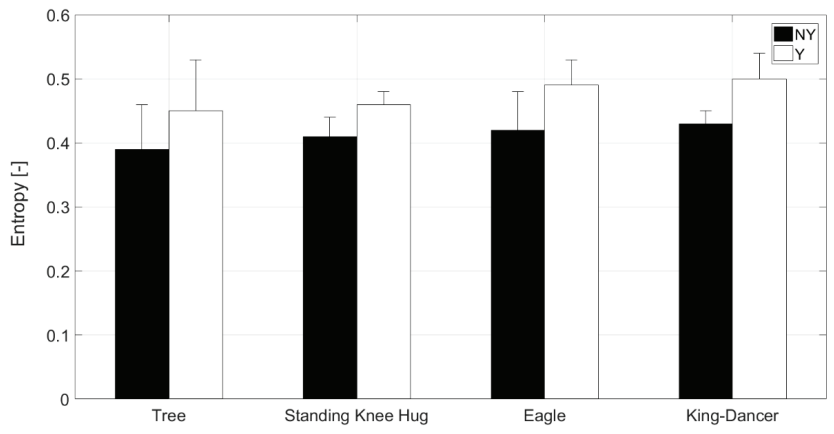

Fig. 2. The mean and standard deviation of entropy values for combined $\mathrm{AP}$ and $\mathrm{ML}$ directions

The maximum mean entropy values in group $\mathrm{Y}$ were $0.5 \pm 0.1$, while in the NY group they were 0.43 \pm 0.06 , for the King-Dancer pose, which constitutes a $14 \%$ difference in entropy values. Minimum values differ only by $13 \%$ (Tree pose).

\section{Discussion}

This objective of the study was to examine CoP regularity in instructors and novices performing four yoga poses, and to verify the additional use of linear and nonlinear methods for assessing postural stability.

Postural control is not a fully automated process [18], but requires a certain level of attention, particularly as the difficulty of the postural task increases. In this paper, the difficulty of the tasks was graded because the yoga poses were ordered from the easiest to the most difficult, which was also confirmed by the results of the CoP path length (Table 3).

The entropy family quantifies signal regularity. A more regular $\mathrm{CoP}$ pattern indicates that the postural behavior is more rigid [4], suggesting a dependency between the regularity of CoP displacements and the amount of attention paid to postural control [4]. On the other hand, complexity is associated with a meaningful structural richness incorporating correlations over multiple spatio-temporal scales. A decrease of complexity is related to functional decline and a more rigid postural behavior in dysfunctional balance control during perturbations [22]. According to this, ballet dancers have high entropy because they do not need to devote much attention to balance, while the elderly have high entropy because they are unable in a similar manner, exert an effective attentive control of balance and "cool-down" the system [2].

In our study, yoga instructors exhibited a larger amount of postural sway (i.e., larger sway area and $\mathrm{CoP}$ path length), suggesting that their balance was overall less stable than that of the non-practicing yoga group. The postural sway of yoga instructors involved more irregular $\mathrm{CoP}$ fluctuations (as exemplified by higher SEn), indicating that their balance was somewhat more automated (demanding less attention) than in novices. This finding is in agreement with Schmit et al. [21], who found that dancers exhibited less regular sway patterns than controls. Schmit et al. [21] also postulated that the increased "noisiness" of postural motions in dancers was indication of greater behavioral flexibility, allowing them to easier switch between behavioral modes. Moreover, in our study, a decrease in SEn (more regular sway fluctuations) was interpreted as a decrease in the effectiveness of postural control. This is in line with the conclusions by Roerdink et al. [18], which stated that healthy physiological systems are often characterized by an irregular and complex type of variability, whereas disease or aging is often associated with greater regularity and less complexity. In summary, statistically significant higher values of SEn were demonstrated in two directions and their combination in the group of yoga instructors. These results indicate that the yoga instructors have an irregular and complex CoP signal, and that they pay less attention when performing individual poses compared to those who don't practice. In addition, we could clearly conclude that the King-Dancer pose had a statistically significantly longer $\mathrm{CoP}$ path length and elliptical field in comparison with other poses. Out of the two groups, this pose also had the highest entropy coefficient. In addition, by arranging the order of the poses in both groups in terms of CoP path length from the shortest to the longest, we obtained the following order: Tree, Standing Knee Hug, Eagle and King-Dancer. In addition to the entropy coefficient, we obtained other measures of nonlinear dynamics: the Lyapunov exponent, the Hurst index and the fractal dimension. The $\lambda$ is assumed as a parameter indexing an orbital instability. Khayat and Nowshiravan-Rahatabad [12] demonstrated that higher $\lambda$ in the 
posture signals of young subjects compared to older ones demonstrate the robustness and responsibility of their control system as a nonlinear complex system. Therefore, a higher $\lambda$ points to the capability of a more rapid response for balance control. A similar interpretation can be applied to this work, because in both groups ( $\mathrm{Y}$ and NY) Lyapunov exponents were the same: 1.06 to 1.11 . Such high $\lambda$ values indicate that a small change in the state of balance causes the control system to show a rapid response to keep the body in a balanced state as the control feedback. Therefore, it can be said that in both groups this mechanism is at a similar level. Analyzing each item individually (Table 3), we observe the most dynamic changes in the postural control response in the King-Dancer pose. Moreover, in all subjects, the $\lambda$ in sagittal plane were larger than those in the medio-lateral direction. In research conducted by Mizuta et al. [14], on prolonged standing and its effect on postural control in elderly individuals in comparison to adults, the medio-lateral direction had a higher $\lambda$. From the mathematical point of view, $\lambda$ were calculated to quantify the exponential separation of nearby trajectories. This information was necessary to assess the stability and determine if the pattern was periodic or chaotic. As nearby points of the state space separate, they diverge rapidly and can produce instability. Lyapunov exponents from a stable system with little to no divergence are equal to zero (sine wave). Alternatively, $\lambda$ for an unstable system that has a high amount of divergence is positive (random data). A chaotic system has both positive and negative $\lambda$, although a positive $\lambda$ indicates instability. These results are additionally confirmed by the Hurst exponent.

The Hurst exponent $(\mathrm{H})$ is a statistical tool for the classification of time series into random and nonrandom series [24]. A Hurst exponent greater than 0.5 indicated that the positive $\lambda$ was due to a persistent chaotic pattern. For both practitioners and non-practitioners of yoga, the Hurst exponents were the same at 0.78 , resulting in a strengthening of the trend, meaning that there is a $78 \%$ probability that the existing trend would be maintained (long-range correlations).

The last parameter to describe chaos in the system is fractal dimension. A fractal pattern describes irregular shapes such as $\mathrm{CoP}$ traces. In the case of yoga instructors in the current cohort, the FD approaches 2 and is significantly higher from these obtained in the novice group, this can be considered to be an indication of a system whose degrees of freedom have been increased to a point where the system is dynamic but under little control, in the case of postural sway the system is swaying equally on either side of the stable position. Higuchi's FD, where this dimension approaches 1 , is indication of a system whose degrees of freedom have been frozen in an attempt to control itself. This is also how the results of this study can be interpreted, where non-practitioners of yoga had lower FD, which means their poses were more frozen (focused).

Researchers consider a FD between 1 and 2 as an indicator of a healthy postural control strategy. When this happens, the participant sways around the stable point in a complex and dynamic manner with a high level of control of the degrees-of-freedom, and as such is able to adapt easily and quickly to changing conditions [5].

\section{Conclusions}

The traditional linear measures of postural sway did not detect a difference between the two groups, but several of the nonlinear measures did detect differences. Therefore, nonlinear measures are more sensitive compared to the traditional parameters and can complement them. Nonlinear measures can provide new insights into the ways the nervous system controls the complexity of balance.

\section{References}

[1] Baratto L., Morasso P.G., Re C., Spada G., A new look at posturographic analysis in the clinical context: sway-density versus other parameterization techniques, Motor Control, 2002, 6 (3), 246-270.

[2] Borg F.G., LAXABACK G., Entropy of balance - some recent results, J. Neuroeng. Rehabil., 2010, 7, 38.

[3] Cavanaugh J.T., Mercer V.S., Stergiou N., Approximate entropy detects the effect of a secondary cognitive task on postural control in healthy young adults: a methodological report, J. Neuroeng. Rehabil., 2007, 4, 42.

[4] Donker S.F., Roerdink M., Greven A.J., Beek P.J., Regularity of center-of-pressure trajectories depends on the amount of attention invested in postural control, Exp. Brain Res., 2007, 181 (1), 1-11.

[5] Doyle T.L., Dugan E.L., Humphries B., Newton R.U., Discriminating between elderly and young using a fractal dimension analysis of centre of pressure, Int. J. Med. Sci., 2004, 1 (1), 11-20.

[6] Doyle T.L., Newton R.U., BuRnetT A.F., Reliability of traditional and fractal dimension measures of quiet stance center of pressure in young, healthy people, Arch. Phys. Med. Rehabil., 2005, 86 (10), 2034-2040.

[7] Gilfriche P., Deschodt-Arsac V., Blons E., Arsac L.M., Frequency-Specific Fractal Analysis of Postural Control Accounts for Control Strategies, Frontiers in Physiology, 2018, 9, 293. 
[8] Goldberger A.L., Amaral L.A., Glass L., Hausdorff J.M., IVAnov P.C., Mark R.G. et al., PhysioBank, PhysioToolkit, and PhysioNet: components of a new research resource for complex physiologic signals, Circulation, 2000, 101 (23), E215-20.

[9] Higuchi T., Approach to an irregular time series on the basis of the fractal theory, Physica D: Nonlinear Phenomena, 1988, 31 (2), 277-283.

[10] HURST H.E., Long-term storage of reservoirs: an experimental study, Transactions of the American Society of Civil Engineers, 1951, 116, 770-799.

[11] JAVNBAKHT M., HeJazi Kenari R., GHASEMI M., Effects of yoga on depression and anxiety of women, Complement Ther. Clin. Pract., 2009, 15 (2), 102-104.

[12] Khayat O., NowshiRavan-Rahatabad F., Complex feature analysis of center of pressure signal for age-related subject classification, Ann. Mil. Health Sci. Res., 2014, 12 (1), 1-6.

[13] MAURER C., PeterkA R.J., A New Interpretation of Spontaneous Sway Measures Based on a Simple Model of Human Postural Control, Journal of Neurophysiology, 2005, 93 (1), 189-200.

[14] Mizuta K., Tokita T., Ito Y., Aoki M., Kuze B., Studies on the largest Lyapunov exponents of the standing posture in patients with unilateral vestibular dysfunction, Nihon Jibiinkoka Gakkai Kaiho, 2009, 112 (12), 791-800.

[15] Pascolo P.B., Marini A., Carniel R., Barazza F., Posture as a chaotic system and an application to the Parkinson's disease, Chaos, Solitons and Fractals, 2005, 24 (5), 1343-1346.

[16] Razjouyan J., Gharibzadeh S., Fallah A., Khayat O., GHERgherehCHI M., AfARIDEH H. et al., A neuro-fuzzy based model for accurate estimation of the lyapunov exponents of an unknown dynamical system, International Journal of Bifurcation and Chaos, 2012, 22 (03), 1250043.
[17] Richman J.S., Moorman J.R., Physiological time-series analysis using approximate entropy and sample entropy, Am. J. Physiol. Heart Circ. Physiol., 2000, 278 (6), H2039-49.

[18] Roerdink M., Hlavackova P., Vuillerme N., Center-ofpressure regularity as a marker for attentional investment in postural control: a comparison between sitting and standing postures, Hum. Mov. Sci., 2011, 30 (2), 203-212.

[19] Rosenstein M.T., Collins J.J., De LuCA C.J., A practical method for calculating largest Lyapunov exponents from small data sets, Physica D: Nonlinear Phenomena, 1993, 65 (1-2), 117-134.

[20] Sabatini A.M., Analysis of postural sway using entropy measures of signal complexity, Medical and Biological Engineering and Computing, 2000, 38 (6), 617-624.

[21] Schmit J.M., Regis D.I., RiLey M.A., Dynamic patterns of postural sway in ballet dancers and track athletes, Exp. Brain Res., 2005, 163 (3), 370-378.

[22] Schniepp R., Wuehr M., Pradhan C., Novozhilov S., KRAFCZYK S., BRANDT T. et al., Nonlinear variability of body sway in patients with phobic postural vertigo, Front. Neurol., 2013, 4, 115.

[23] SHIRAISHI J.C., BeZERRA L.M.A., Effects of yoga practice on muscular endurance in young women, Complementary Therapies in Clinical Practice, 2016, 22, 69-73.

[24] TARNOPOLSKI M., On the relationship between the Hurst exponent, the ratio of the mean square successive difference to the variance, and the number of turning points, Physica A: Statistical Mechanics and its Applications, 2016, 461(C), 662-673.

[25] Ulger O., YAGLi N.V., Effects of yoga on balance and gait properties in women with musculoskeletal problems: a pilot study, Complement Ther. Clin. Pract., 2011, 17 (1), 13-15. 